\title{
Morphology and Electronic Properties of Hybrid Organic-Inorganic System: Ag Nanoparticles Embedded into CuPc Matrix
}

\author{
I. M. Aristova ${ }^{1}$, O.Yu.Vilkov ${ }^{2}$, A. Pietzsch ${ }^{3}$, M. Tchaplyguine ${ }^{3}$, O.V. Molodtsova ${ }^{4}$, V.Yu. Aristov ${ }^{1,5}$ \\ ${ }^{1}$ Institute of Solid State Physics, Russian Academy of Sciences, Chernogolovka, Russia \\ ${ }^{2}$ Helmholtz Zentrum Berlin Mat\&Energy, Berlin, Germany \\ ${ }^{3}$ Lund University, MAX-lab, Lund, Sweden \\ ${ }^{4}$ Hasylab, Desy, Hamburg, Germany \\ ${ }^{5}$ European XFEL GmbH, Hamburg, Germany
}

Received 2012

\begin{abstract}
Materials with a high on-off resistance ratio could become the basis for resistive random-access memory (RRAM). It is assumed that one of RRAM types can be based on hybrid organic-inorganic systems, while particular attention is focused on hybrid systems consisting of metal nanoparticles (NP) embedded in organic matrix (OM). In this investigation we created and studied the hybrid organic-inorganic systems made of metal (Ag) nanoparticles embedded in organic semiconductor material CuPc. The LEED patterns and NEXAFS data demonstrate that the CuPc films deposited on Au(001) substrate are highly ordered and molecular planes lie parallel to the gold surface. The metal atoms were deposited on the outer surface of the organic molecular film and self-assembled into nanoparticles due to surface and bulk diffusion. The properties of nano-composite materials seem to be significantly dependent on the microstructure, i.e. the size, concentration, bulk- and size-distribution of nanoparticles; therefore we have studied by high resolution transmission electron microscopy the evolution of morphology of nano-composite films as a function of nominal metal deposition. The filled and empty electronic states of the hybrid organic-inorganic systems, energy level alignment at interfaces formed between metal nanoparticles and the organic semiconductor CuPc as well as the chemical interaction at the NP/OM interface were studied by UPS, XPS and NEXAFS methods.
\end{abstract}

Keywords: Hybrid Materials; Nanoparticles; Organic Matrix; Morphology; Electronic Properties; Nonvolatile Memory

Nowadays there are tremendous worldwide efforts to develop new memory devices for long-term storage of the information $[1,2]$. The new kind of memory based on switchable resistive materials is commonly ranked as resistive random-access memory (RRAM). Hybrid systems, mainly consisting of inorganic nanoparticles (NP) blended into an organic matrix have been proposed as one type of RRAM [3,4]. Possible memory architecture is obtained if an organic thin film containing metal nanoparticls is sandwiched between cross-point arrays of electrodes - that consist of narrow metal stripes, running in perpendicular directions above and below the film (see Figure 1).

The resistivity at "cross-points" can be switched either a high- or low-conductivity state by applying a voltage, suitable to write or erase.

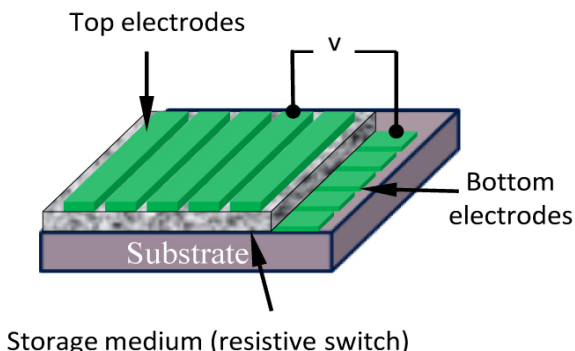

Figure 1. Cross-point memory architecture
Above a threshold voltage the device suddenly switches from a high-impedance state to a low-impedance state and remains in that state even when the power is off. The resistivity in the high- and low conductivity state can differ by 6-8 orders of magnitude [5]. The high-impedance state can be recovered by applying a voltage in the reverse direction. The corresponding state can then be probed by measuring the current across the crosspoints at some lower voltages. By defining the two states it is possible to create digital memory devices. These two states can be viewed as the realization of non-volatile electrical memory, thus rendering the structures suitable for data storage.

Albeit, there is a rapid development in this area but the precise memory mechanism is still unclear and many questions left unanswered [6-8]. Properties of the materials formed with participation of nanometer-sized (tens or units nanometers) structural elements, are not identical with the properties of bulk matter, so it is possible to consider such nanostructures as a special state of substance. The properties of such composite films are strongly linked to the particles' nanostructure, i.e. the size, concentration, bulk- and size-distribution of respective nanoparticles. The most fertile model for mechanism that is responsible for the large difference in resistivity between the high and low conductivity states is based on charging of the NP, which leads to strong modifications of the properties of the organic matrix material (see Review [5] and references therein). 
It is impossible to understand the underlying mechanism without comprehensive information about the specific electron structure and interface interactions between the NP's and the thin organic film matrix. The aim of the present work was to investigate the diverse morphologically defined hybrid systems composed of silver NP's (Ag NP's) distributed in a CuPc (organic semiconductor copper phthalocyanine) matrix, which is formed by molecules with a wide energy gap and delocalized $\pi$-orbitals.

The organic semiconductor copper phthalocyanine matrix, CuPc thin film, for hybrid organic-inorganic system was grown on an atomically clean $\mathrm{Au}$ (001) surface by deposition of organic molecules evaporated from an effusion cell under ultrahighvacuum (UHV) conditions.

No residual contamination was detected in core-level photoemission (CL-PES) spectra. Morphology and ordering of the CuPc overlayer were evaluated by means of LEED and nearedge $\mathrm{x}$-ray absorption fine structure spectroscopy (NEXAFS) $[9,10]$, which demonstrate that the CuPc films are highly ordered and molecular planes lie parallel to the gold substrate. Figure 2 reveal a very strong angular dependence of the $\mathrm{N} 1 \mathrm{~s}-$ $\pi^{*}$ intensities (396-404 eV). At grazing incidence, when polarization vector $\mathbf{E}$ is almost perpendicular to the subbstrate, the intensities of these $\pi^{*}$ signals show a maximum, while reaching a minimum, when $\mathbf{E}$ is almost parallel to the substrate. The angular dependence of the $\mathrm{N} 1 \mathrm{~s}-\sigma^{*}$ intensities $(404-420 \mathrm{eV})$ reveals the opposite trend. This behavior together with registered LEED patterns demonstrates, that the CuPc molecules are well ordered with the molecular planes lying parallel to the Au (001) substrate. This observation proves that the $\mathrm{CuPc}-\mathrm{Au}(001)$ substrate coupling is stronger than the $\mathrm{CuPc}-\mathrm{CuPc}$ interaction.

Resistive evaporation of a high-purity silver wire wound around a thoroughly degassed tungsten filament was used for metal deposition on the outer surface of the organic film. Due to surface and bulk diffusion of deposited Ag atoms the embedded silver nanoparticles were self-assembled forming a three-dimensional Ag NP's distribution in the bulk of the organic semiconductor.

The size, concentration, size-distribution and shape of the resulting nanoparticles were studied using transmission electron microscopy (TEM) JEOL JEM-2100 operated at $200 \mathrm{kV}$. Thus the microstructure and evolution of the morphology of the nano-composite films as a function of nominal silver coverage were studied (see Figures 3 and $\mathbf{4}$ ).

The hybrid organic (CuPc) - inorganic (Ag) systems for TEM investigations were prepared under UHV conditions on cleaved $\mathrm{NaCl}$ single crystals as substrate using the same UHV chamber, evaporators and the same parameters (temperature, rate of CuPc deposition, organic film thickness, rate of silver deposition, nominal silver coverage, etc.) as for the systems grown on $\mathrm{Au}(001)$ surface. In order to bind the nano-composite thin films these samples were then coated by an ultra thin overlayer of amorphous carbon. Film separation from a substrate always occurs easily by dissolution salt in water. Preliminary the film was cut by square pieces with the side of about 2-3 mm. Then samples are transferred on 250-mesh (250 lines/inch) copper grids for TEM investigations.

Figures 3-4 presents bright-field TEM images of nanocomposite thin films microstructure. It composed of silver particles (the black dots in the picture) embedded in an organic semiconductor matrix CuPc for nominal Ag deposition of 0.4 and $5.7 \mathrm{~nm}$. The electron diffraction patterns for selected areas of the samples with corresponding silver deposition prove that the diffraction originates from silver NP's. Figures 3-4 demonstrate that the size of the Ag NP's strongly depends on silver coverage. For nominal Ag deposition of $0.4 \mathrm{~nm}$ (Figure 3) silver nanoparticles form some particle distribution, while metal nanoparticles are spherical. With further deposition one observes strong growth of individual grains. Large particles have an irregular shape. The analysis of size distribution of silver nanoparticles allows to assume the following: due to increase of nominal coverage of silver some nearby nanoparticles (because of increase their sizes) come to contact and coalesce, forming larger particles.

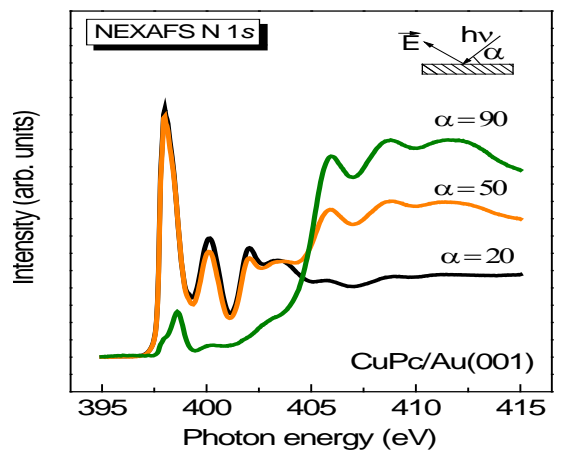

Figure 2. N 1s NEXAFS spectra taken from CuPc film (7 $\mathrm{nm}$ thick), deposited on the $\mathrm{Au}(001)$ surface, taken at 3 different angles alpha between the light polarization vector $E$ and the normal to the sample surface $n$. The inset indicates the experimental geometry. The lower energy features $(396-404 \mathrm{eV})$ represent the $\pi^{*}$ resonances, whereas the features above $404 \mathrm{eV}$ are related to the $\sigma^{*}$ resonances.

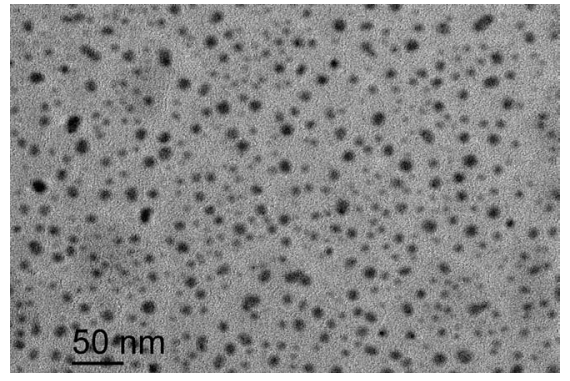

(a)

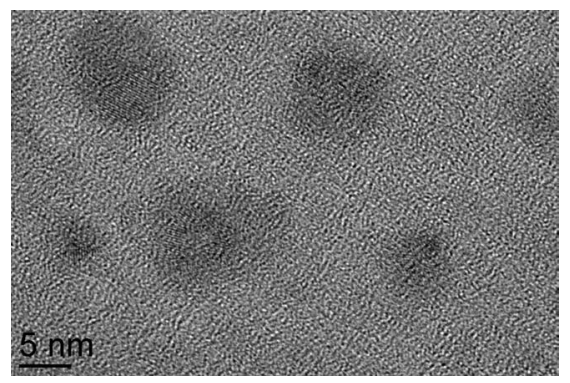

(b)

Figure 3. Microstructure of nanocomposite thin film composed of silver particles embedded in CuPc matrix (TEM) for 2 different (a and b) magnifications. Nominal Ag deposition is $0.4 \mathrm{~nm}$. 


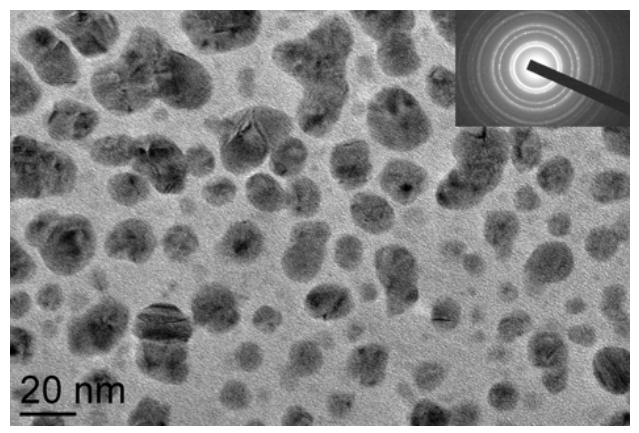

(a)

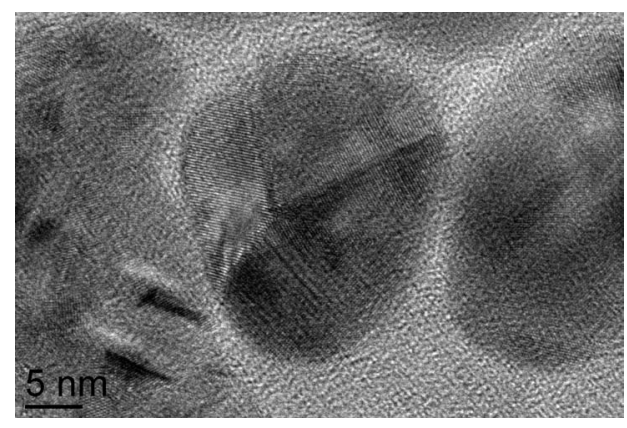

(b)

Figure 4. Microstructure of nanocomposite thin film composed of silver particles embedded in CuPc matrix (TEM) for 2 different (a and b) magnifications. Nominal Ag deposition is $5.7 \mathrm{~nm}$.

Coalescence of two or more small particles in one large particle leads to reduction of particles surface area and therefore to reduction of particles surface energy. Thus, we observe the coalescence process of silver nanoparticles embedded in the $\mathrm{CuPc}$ film surface. It leads to increase of average diameter of the particles and thereby has an influence on size distribution of silver nanoparticles.

The electronic structure (occupied and unoccupied states) of the hybrid organic-inorganic systems, the energy level alignment at interfaces formed between Ag NP's and the organic semiconductor $\mathrm{CuPc}$, as well as the chemical interaction at this interface were studied by CL-PES, valence band PES (VB-PES) and NEXAFS methods. The work function (WF) changes induced by silver deposition on CuPc thin films were determined from VB-PES data using the cut-off procedure. All PES and
NEXAFS measurements were performed at BESSY (Berlin) and MAX-Lab (Lund).

\section{Acknowledgements}

This work was supported by the RFBR under grant no. 10-02-00269 and grant no. 11-02-01253.

\section{REFERENCES}

[1] Z. Liu, A.A. Yasseri, J.S. Lindsey, D.F. Bocian, Molecular Memories That Survive Silicon Device Processing and Real-World Operation, Science, vol. 302, 2003, pp. 1543-1545.

[2] J.C. Scott, Is There an Immortal Memory?, Science, vol. 304, 2004, pp. 62-63; J.C. Scott and L.D. Bozano, Nonvolatile Memory Elements Based on Organic Materials, Adv. Mater., vol. 19, 2007, pp. 1452-1463.

[3] L.D. Bozano, B.W. Kenan, M. Beinhoff, K.R. Carter, P.M. Rice, J.C. Scott, Organic materials and thin-film structures for cross-point memory cells based on trapping in metallic nanoparticles, Adv. Funct. Mater., vol. 15, 2005, pp. 1933-1939.

[4] Y.Yang, J.Ouyang, L. Ma, R.J.-H. Tseng, C.-W. Chu, Electrical Switching and Bistability in Organic/Polymeric Thin Films and Memory Devices Adv. Funct. Mater., vol. 16, 2006, pp. 1001-1014.

[5] D. Prime, S. Paul, and P.W. Josephs-Franks, Gold nanoparticle charge trapping and relation to organic polymer memory devices, Phil. Trans. R. Soc., vol. A367, 2009, pp. 4215-4225.

[6] J.Y. Ouyang, C.W. Chu, C.R. Szmanda, L. Ma and Y. Yang, Programmable polymer thin film and non-volatile memory device, Nat. Mater., vol. 3, 2004, pp. 918-922.

[7] D. Tondelier, K. Lmimoumi, C. Fery, and G. Haas, Metal/ organic/metal bistable memory devices, Appl. Phys. Lett., vol. 85, 2004, pp. 5763-5765.

[8] L. D. Bozano, B. W. Kenan, V. R. Deline, J. R. Salem, and J. C. Scott, Mechanism for bistability in organic memory elements, Appl. Phys. Lett., vol. 84, 2004, pp. 607-609.

[9] H. Peisert, T. Schwieger, J. M. Auerhammer, M. Knupfer, M. S. Golden, J. Fink, P. R. Bressler, and M. Mastet al., Order on disorder: Copper phthalocyanine thin films on technical substrates, J. Appl. Phys., vol. 90, 2001, pp. 466-469.

[10] G. Cabailh, J. W. Wells, I. T. McGovern, A. R. Vearey-Roberts, A. Bushell, and D. A. Evans Synchrotron radiation studies of the growth and beam damage of tin-phthalocyanine on GaAs (001)-1x6 substrates, Appl. Surf. Sci., vol. 234, 2004, pp. 144-148. 